\title{
Perfil de Utilização dos Serviços Odontológicos na Atenção Primária à Saúde: Experiência de Duas Unidades de Saúde do Município de Porto Alegre - Rio Grande do Sul
}

Oral Health Care in Primary Care Attention: Experience from Two Facilities of Porto Alegre - Rio Grande do Sul

Gustavo Giacomelli Nascimento ${ }^{1}$, Caroline Machado Weber ${ }^{2}$, Daniel Demétrio Faustino da Silva ${ }^{3}$

\section{Abstract}

Aim: Describe the dental service utilization profile from US NSA and US SESC - Grupo Hospitalar Conceição.

Materials and methods: The study evaluated a Sistema de Informações -SIS' report obtained from dental appointments (June and August 2011. The variables were: demographic (age, gender and territory), aim of appointment (ICD-10) and type of it (booked or urgent).

Results: Most patients from US NSA were women, age 35-44 years, coming from the area NSA3; seeking for booked appointments for treating dental caries. At US SESC, most patients were women, age 0-11 years, from the area SESC2, with a greater rate of booked appointments and utilization for complete dental exam/dental appointment to children 0-3 years.

Conclusion: The results show that increasing the use of SIS data for Dentistry can support the upgrading of resource distribution and management of oral health care in accordance with the principles of Primary Care Attention.

Keywords: Primary Care Attention, access to care, utilization, information systems, oral health.

\section{Resumo}

Objetivo: estabelecer o perfil de utilização dos serviços odontológicos nas Unidades de Saúde SESC e NSA do Grupo Hospitalar Conceição.

Materiais e métodos: analisou-se o relatório do Sistema de Informação-SIS das consultas odontológicas (meses junho a agosto/2011). As variáveis foram: características sócio-demográficas (idade, gênero e microárea), motivo da consulta (CID-10) e tipo de consulta ambulatorial: consulta programada ou consulta não programada.

Resultados: na US NSA prevaleceu os usuários do gênero feminino, faixa etária 35-44 anos, proveniente da microárea NSA3; o acesso aos serviços odontológicos foi por consultas programadas, e o código CID-10 de cárie dentária foi o mais freqüente. Na US SESC destacase de diferente a faixa etária de 0-11 anos e maior utilização do código CID-10 de primeira consulta odontológica programada.

Conclusão: Os achados demonstram que é possível utilizar os dados do SIS para conhecer o perfil de utilização e a partir destes organizar o acesso em SB, baseado nos princípios APS.

Palavras-chave: Atenção Primária à Saúde, acesso aos serviços de saúde, utilização, sistemas de informação, saúde bucal.
'Odontólogo Residente. Residência Integrada em Saúde, Ênfase em Saúde da Família e Comunidade, Grupo Hospitalar Conceição, RS, Brasil. Doutorando em Odontologia - Programa de Pós-graduação em Odontologia, UFPel.

${ }^{2}$ Odontóloga Residente. Residência Integrada em Saúde, Ênfase em Saúde da Família e Comunidade, Grupo Hospitalar Conceição, RS, Brasil.

${ }^{3}$ Odontólogo do Serviço de Saúde Comunitária. Grupo Hospitalar Conceição, RS, Brasil. Mestre em Clínica Odontológica-Odontopediatria e Doutorando em Saúde Bucal Coletiva - Programa de Pós-graduação em Odontologia, UFRGS.

Correspondência: Daniel Demétrio Faustino da Silva

Endereço: Grupo Hospitalar Conceição

Serviço de Saúde Comunitária - Unidade de Saúde SESC

Rua Ernesto Pellanda, 830, Vila Jardim - CEP 91320-220, Rio Grande do Sul Brasil

Fone: (51) 33348383

E-mail: ddemetrio@gmail.com

Data de Submissão: 10407/2012

Data de Aceite: 30/08/2012

\section{Introdução}

O Serviço de Saúde Comunitária (SSC) do Grupo Hospitalar Conceição (GHC) compõe-se de 12 Unidades de Saúde (US) localizadas na Zona Norte do município de Porto Alegre - Rio Grande do Sul, com todos os seus atendimentos realizados pelo Sistema Único de Saúde (SUS). Suas equipes multiprofissionais são compostas por médicos de família e comunidade, cirurgiões-dentistas e enfermeiros especialistas em saúde coletiva, psicólogos, assistentes sociais, técnicos e auxiliares de enfermagem, técnicos em saúde bucal, auxiliares administrativos e agentes comunitários de saúde, além de nutricionistas e farmacêuticos, todos trabalhando de acordo com os princípios da Atenção Primária em Saúde (APS) no contexto do SUS. Além disso, as unidades do SSC são campos de formação tanto para estagiários de diversos cursos de graduação de universidades conveniadas como para residentes dos programas de Residência Integrada em Saúde e de Residência em Medicina de Família e Comunidade. Ainda que pertençam ao mesmo Serviço, cada unidade possui suas especificidades, principalmente no que se refere às características do território, às equipes e ao seu processo de trabalho. No entanto, algumas delas compartilham características semelhantes, como a Unidade de Saúde Nossa Senhora Aparecida (US NSA) e a Unidade de Saúde SESC (US SESC).

Para organizar o Serviço e proporcionar que as equipes de saúde trabalhem segundo a lógica da vigilância em saúde, o SSC implementou, nas unidades de saúde, o Sistema de Informações em Saúde (SIS), desenvolvido pelo GHC, adaptado às necessidades das unidades e em consonância com o Sistema de Informações do município de Porto Alegre, por meio de um Núcleo de Monitoramento e Avaliação. Esse sistema é alimentado sistematicamente com dados referentes ao usuário (idade, gênero e local de moradia), à assistência e a atividades de promoção de saúde, com os códigos do 
Sistema de Informações Ambulatoriais do Sistema Único de Saúde (SIA-SUS) e da Classificação Estatística Internacional de Doenças e Problemas Relacionados à Saúde - Décima Revisão (CID-10). Estas informações, traduzidas por meio de relatórios, servem como base para o trabalho de vigilância em saúde, lógica tão importante para a APS, uma vez que, por meio deles, tenta-se contemplar os princípios deste modelo de atenção: acesso, longitudinalidade, integralidade e coordenação do cuidado.

Segundo Andersen (1995) e Starfield (2002), o acesso aos serviços de saúde traz consigo outros conceitos, e contempla não só a entrada nos serviços (acessibilidade), mas também o recebimento de cuidados subsequentes (utilização). Para Pereira (2002), o acesso à saúde envolve aspectos de ordem socioeconômica e cultural, que extrapolam a assistência em saúde e tem sido considerado um fator de grande importância na utilização de serviços de saúde. O padrão de utilização tem sido organizado a partir da demanda por serviços de saúde, voltando-se principalmente para o perfil de necessidades individuais, e também para características demográficas do território (SAWEYR; LEITE; ALEXANDRINO, 2002).

Para aprofundar o entendimento sobre a utilização dos serviços de saúde, é necessária a substituição do enfoque de doença pelo enfoque território-população. $O$ reconhecimento desse território pelos profissionais de saúde é uma etapa fundamental para a caracterização da população e de seus problemas e necessidades de saúde, permitindo a identificação de vulnerabilidades, de populações expostas e de problemas prioritários para as intervenções, bem como a avaliação do impacto dos serviços sobre os níveis de saúde desta população (GONDIM et al., 2008). Dessa forma, o ponto de partida para a organização das ações de vigilância em saúde é a territorialização do sistema local de saúde, que consiste em compreender o território segundo a lógica das relações de vida, ambiente e acesso às ações e serviços de saúde. A partir disso, torna-se possível identificar subdivisões em pequenas extensões dos territórios - denominadas micro áreas -, nas quais seus habitantes possuem uma condição de vida homogênea. As micro áreas podem ser consideradas de risco pelas equipes de saúde quando se identificam moradores ou famílias mais expostas aos riscos de adoecer e morrer, ou onde as condições de vida e a situação de saúde são precárias (MALUCELLI et al., 2010).

Entendendo a saúde bucal como parte integrante desse novo processo, a incorporação do cirurgião-dentista na APS tem sido vista como uma possibilidade de se romper com os modelos assistenciais em saúde bucal excludentes, baseados em curativismo, tecnicismo e biologicismo. Isso porque a APS, de certa forma, tenta modificar a lógica programática desses modelos, já que não só articula as propostas da vigilância à saúde baseando-se na integralidade, como também possui como um de seus princípios a busca ativa de famílias, as quais são consideradas como núcleo social primário (SOUZA; RONCALLI, 2007). Apesar da reconhecida importância da saúde bucal, uma parcela importante aproximadamente $12 \%$ da população brasileira - nunca foi ao dentista e/ou ainda não tem acesso aos serviços de saúde (INSTITUTO BRASILEIRO DE GEOGRAFIA E ESTATÍSTICA, 2010). Por isso mesmo, um dos objetivos dos serviços públicos odontológicos é reduzir os efeitos das desigualdades sociais hoje verificadas em relação à saúde bucal (MATOS et al., 2002).

O objetivo deste trabalho foi estabelecer o perfil de utilização dos serviços odontológicos nas US NSA e US SESC com base nos relatórios do SIS.

\section{Materiais e Métodos}

Este trabalho foi submetido e aprovado pelo Comitê de Ética em Pesquisa do Grupo Hospitalar Conceição.

O estudo foi realizado nas US NSA e US SESC do SSC do GHC, situadas nos distritos leste-nordeste e norte-eixo Baltazar do município Porto Alegre, Rio Grande do Sul, Brasil. A US NSA fica localizada no bairro Sarandi e possui população cadastrada de aproximadamente cinco mil habitantes, tendo seu território dividido em três micro áreas. A US SESC fica localizada no bairro Vila Jardim e possui uma população cadastrada de aproximadamente seis mil habitantes, com território adscrito dividido em cinco micro áreas. A divisão do território deu-se para facilitar o trabalho de vigilância em saúde de acordo com critérios específicos de cada unidade de saúde. Ambas as unidades contam com núcleo de Odontologia formado por três cirurgiões-dentistas contratados, um técnico em saúde bucal e dois cirurgiões-dentistas residentes, sendo um de primeiro e um de segundo ano. O acesso à Odontologia na US NSA se dá de duas maneiras: consultas programadas por meio de agendamento semanal e consultas não programadas por meio do acolhimento, em que uma dupla de diferentes núcleos profissionais escuta, discute e avalia a necessidade de encaminhamento à equipe de saúde bucal. Na US SESC, o agendamento de consultas programadas se dá por meio do Grupo de Acesso em Saúde Bucal realizado quinzenalmente, que consiste em atividade educativa e triagem com classificação de risco; as consultas não programadas são avaliadas exclusivamente pela equipe de saúde bucal a partir da queixa do paciente aos profissionais administrativos.

Foi analisado o relatório do SIS referente a consultas odontológicas realizadas por cirurgiões-dentistas contratados e residentes entre os meses de junho a agosto de 2011, somando 1206 boletins entre as duas US. As variáveis analisadas foram: características sócio-demográficas (idade, gênero e micro área), motivo da consulta (CID-10) e tipo de consulta ambulatorial SIASUS: consulta programada (0301010153/0301010030) ou consulta não programada (0301060037). Não foram incluídos os boletins de atendimento gerados a partir de consultas odontológicas de pacientes não pertencentes às áreas de abrangências das unidades de saúde, bem como aqueles gerados a partir de atividades não ambulatoriais (atividades coletivas e visitas domiciliares).

Previamente ao período de coleta de dados, foi realizada oficina de treinamento com os cirurgiões-dentistas contratados e residentes das US participantes do estudo, a fim de uniformizar o preenchimento dos boletins de atendimento. $O$ treinamento dos profissionais se deu por meio de casos clínicos, utilizando-se a listagem resumida da CID-10, com base em estudo conduzido no SSC (RODRIGUES; FERNANDEZ; FUNK, 2010) que contempla as doenças odontológicas mais prevalentes nos serviços de saúde e em listagem dos códigos SIASUS existentes para os procedimentos odontológicos.

A análise dos dados foi realizada com auxílio do software SPSS, versão 17.0, para Windows. Foi utilizado o teste qui-quadrado para as variáveis independentes (gênero e faixa etária) entre as unidades e a associação das variáveis gênero, faixa etária, micro área e CID-10 e a variável tipo de consulta em cada unidade. O nível de significância foi de $5 \%$.

O projeto foi aprovado pelo Comitê de Ética em Pesquisa do Grupo Hospitalar Conceição. 


\section{Resultados}

No período de junho a agosto de 2011, foram gerados 1206 boletins de atendimento para consultas odontológicas nas duas unidades de saúde, sendo 773 (64\%) na US NSA e 433 (36\%) na US SESC. A Tabela 1 mostra a distribuição dos pacientes atendidos de acordo com gênero, faixa etária e micro área de vigilância do território das unidades de saúde. Não foram observadas diferenças estatisticamente significativas entre as unidades com relação à variável gênero. Porém, em ambas as unidades houve uma maior frequência de utilização dos serviços odontológicos pelo gênero feminino. Na US NSA, a média de idade dos pacientes atendidos foi de 30,6 anos (idade de $0-80$ ) e na US SESC, foi de 26,8 anos (idade de 0-88). Quando se comparou as duas unidades, observou-se diferença estatisticamente significativa em relação ao número de atendimentos na faixa etária de 35-44 anos na US NSA e na de 0-11 anos na US SESC $(p<0,05)$. As micro áreas NSA3 e SESC2, da US NSA e US SESC, respectivamente, foram as que concentraram 0 maior número de pacientes atendidos.

Tabela 1. Distribuição das variáveis independentes nas US NSA e US SESC. Número e (porcentagem).

\begin{tabular}{lllll}
\hline & \multicolumn{2}{c}{ US NSA, $\mathbf{n}(\%)$} & & $\begin{array}{c}\text { US SESC, } \mathbf{n} \\
\text { Variável }\end{array}$ \\
SEXO & & $\mathrm{P}$ & & $\mathrm{P}$ \\
\hline & $\mathrm{M}$ & $157(40,1)$ & & $120(42,7)$ \\
& $\mathrm{F}$ & $235(59,9)$ & & $161(57,3)$ \\
& Total & $392(100,0)$ & & $281(100,0)$
\end{tabular}

\section{FAIXA ETÁRIA}

$\begin{array}{llc}0-11 & 84(21,4) & 96(34,2)^{*} \\ \text { dez/18 } & 41(10,5) & 22(7,8) \\ 19-34 & 113(28,8) & 72(25,6) \\ 35-59 & 125(31,9)^{*} & 68(24,2) \\ 60 \quad+ & \\ \text { anos } & 29(7,4) & 23(8,2) \\ \text { Total } & 392(100,0) & 281(100,0)\end{array}$

\section{MICROÁREAS}

\begin{tabular}{lccc} 
nsa1 & $120(30,6)$ & sesc1 & $81(28,8)$ \\
nsa2 & $130(33,2)$ & sesc2 & $89(31,7)$ \\
nsa3 & $142(36,2)$ & sesc3 & $73(26,0)$ \\
& & sesc4 & $21(7,5)$ \\
& & sesc5 & $17(6,0)$ \\
Total & $392(100,0)$ & & $281(100,0)$ \\
\hline
\end{tabular}

*Diferença estatística (teste qui-quadrado, $\mathrm{P}<0,05$ )

A Tabela 2 evidencia a distribuição das consultas quanto ao tipo (programada e não programada) por US. Nota-se diferença entre o número de boletins e o número de consultas devido ao não preenchimento do código SIASUS referente ao tipo de consulta em 34 boletins da US NSA e em um boletim da US SESC.
Tabela 2. Distribuição dos tipos de consulta na US NSA e US SESC. Número e (porcentagem).

\begin{tabular}{llll} 
US & $\mathrm{P}, \mathrm{n}(\%)$ & $\mathrm{NP}, \mathrm{n}(\%)$ & Total \\
\hline & & & \\
NSA & $659(89,2)$ & $80(10,8)$ & 739 \\
SESC & $369(85,4)$ & $63(14,6)$ & 432 \\
\hline
\end{tabular}

$\mathrm{P}$, consulta programada

NP, consulta não programada

Os códigos CID-10 mais utilizados pelos profissionais estão descritos nas Figuras 1 e 2. Na US NSA, os códigos CID-10 mais utilizados foram K02 - cárie dentária (21,9\%), K04 - doenças da polpa dentária e dos tecidos periapicais (19,3\%) e Z01.2 - exame odontológico completo/consulta da ação programática odontológica de 0-3 anos (14,5\%); na US SESC, foram Z01.2 (27,3\%), K02 $(24,7 \%)$ e K04 (18,2\%).

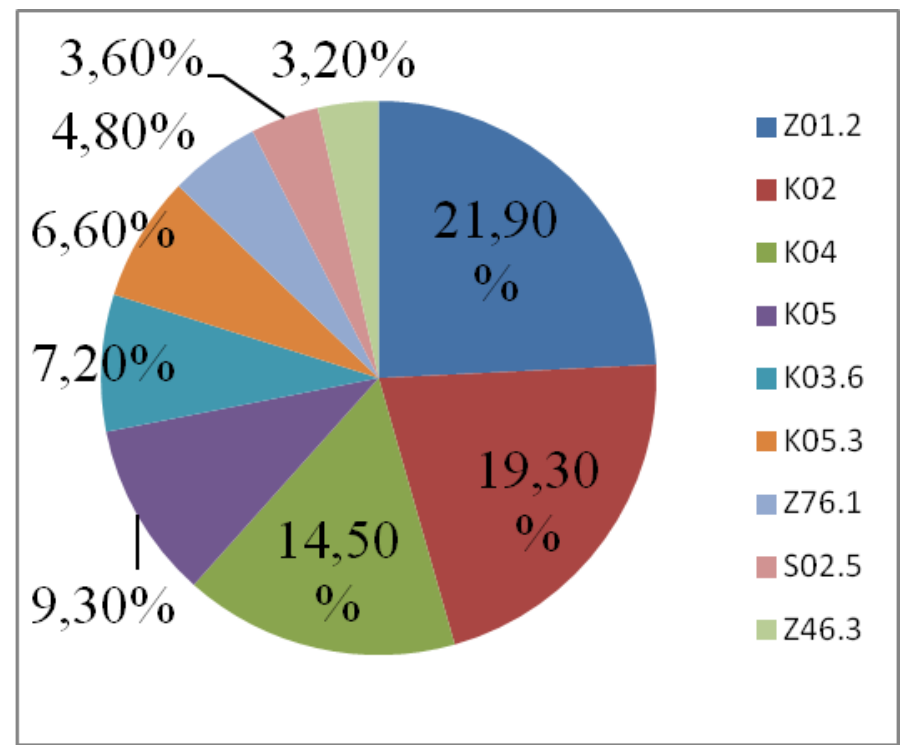

Figura 1. Códigos CID-10 mais utilizados, em valores percentuais na US NSA

Códigos CID-10: Z01.2: Primeira consulta odontológica anual; K02: cárie dentária; K04: doença da polpa e dos tecidos periapicais; K05: gengivite e doenças periodontais; K03.6: depósito nos dentes; K05.3: periodontite crônica; Z76.1: fratura de restauração; S02.5: fratura de dentes; Z46.3: colocação e ajuste de prótese dentária. 


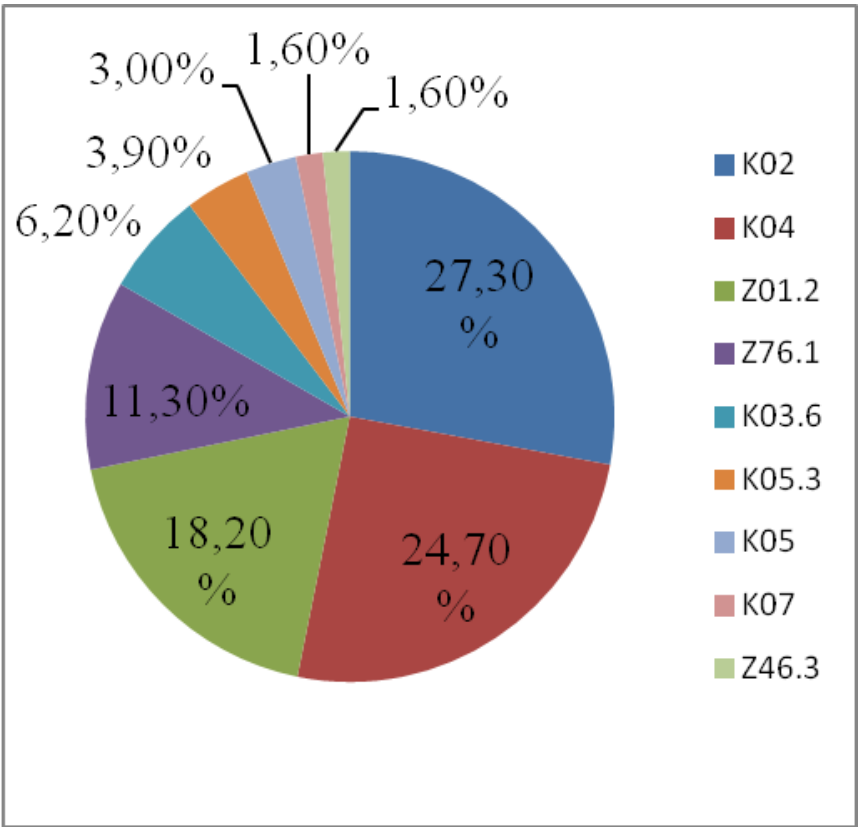

Figura 2. Códigos CID-10 mais utilizados, em valores percentuais na US SESC.

Códigos CID-10: K02: cárie dentária; K04: doença da polpa e dos tecidos periapicais; Z01.2: Primeira consulta odontológica anual; Z76.1: fratura de restauração; K03.6: depósito nos dentes; K05.3: periodontite crônica; K05: gengivite e doenças periodontais; K07: Anomalias dentofacias (maloclusão); Z46.3: colocação e ajuste de prótese dentária.

Quando se analisou a associação entre a variável tipo de consulta com as variáveis gênero, faixa etária e micro área, apenas se evidenciou diferença estatisticamente significativa na US NSA quanto ao tipo de consulta e sua distribuição nas micro áreas, predominando na área NSA1 as consultas não programadas (43,8\%) e na área NSA3 as consultas programadas $(37,0 \%)(P<0,05)$.

Houve associação entre 0 código K02 e consultas programadas na US NSA e entre o código K04 e consultas não programadas em ambas as unidades $(P<0,05)$.

\section{Discussão}

A APS é um modelo de atenção à saúde que objetiva atender ao indivíduo e à família de forma integral e contínua, desenvolvendo ações de promoção, proteção e recuperação da saúde, coordenando os cuidados necessários ao paciente e fazendo a regulação entre os níveis de atenção primária, secundária, terciária e entre os diversos setores relacionados à saúde (BRASIL, 1997). Pretende ser ela, portanto, a porta de entrada do cidadão ao sistema de saúde, prestando serviços mais resolutivos, integrais e, principalmente, humanizados (LEVCOVITZ; GARRIDO, 1996).

No entanto, essa nova visão da atuação na saúde, com uma equipe de saúde da família que considera a integralidade da assistência e da prevenção e trata o indivíduo como sujeito dentro da sua comunidade socioeconômica e cultural, somente teve oficialmente incorporada a figura do cirurgião-dentista em 2000, em um contexto político, econômico e social favorável. Até então, a prestação de serviços de saúde bucal no Brasil caracterizava-se por ações de baixa complexidade, predominantemente curativas e mutiladoras, com acesso restrito, e a maioria dos municípios brasileiros desenvolvia ações voltadas para a faixa etária escolar, ofertando para adultos e idosos apenas serviços de urgência, geralmente mutiladores. Por essas razões, a Odontologia era considerada uma das áreas da saúde com maior exclusão social, menos equânime e universal (A POLÍTICA..., 2011).

Os resultados do presente estudo evidenciam que, apesar das especificidades de cada US, há semelhanças no perfil de acesso no que se refere às variáveis sócio-demográficas. A frequência de pacientes dos gêneros feminino e masculino foi similar, com maior utilização dos serviços odontológicos por pacientes do gênero feminino em ambas as US. Há uma tendência na literatura que aponta para uma maior utilização dos serviços de saúde, de um modo geral, pelas mulheres do que pelos homens (INSTITUTO BRASILEIRO DE GEOGRAFIA E ESTATÍSTICA, 2010; PINHEIRO et al., 2002; BARROS; BERTOLDI, 2002). Essa observação pode ser explicada pelas variações tanto no perfil de necessidades de saúde entre os gêneros como pelo maior interesse das mulheres com relação à sua saúde. Ainda, verificou-se que as mulheres utilizavam com maior regularidade um mesmo serviço de saúde, quando comparadas com os homens (VERBRUGGE, 1989).

Desde o ano de 2009, o SSC incorporou ao Programa da Criança uma ação de vigilância em saúde bucal, com o objetivo de diminuir a prevalência de cárie precoce na infância. A ação, que se iniciou em 2010, prevê uma consulta anual até que a criança complete três anos de idade; para isso, o acesso à Odontologia é livre. Os esforços destinados ao atendimento de crianças na faixa etária de 0-3 anos, principalmente nos meses de junho e agosto de 2011, em paralelo com as atividades da Campanha Nacional de Vacinação contra a Poliomielite, podem explicar os achados na US SESC, em que se observou maior frequência de pacientes atendidos na faixa etária de 0 a 11 anos. Isso pode justificar, também, o maior uso do código de primeira consulta odontológica/consulta da ação programática odontológica de 0-3 anos naquela US. Alguns estudos que avaliaram a utilização de serviços odontológicos entre crianças/jovens, adultos e idosos indicaram taxas mais elevadas de ida ao dentista nos indivíduos em idade escolar, decrescendo a partir dos 15 anos (PINHEIRO et al., 2002). Deve-se ressaltar que as crianças estão entre aquelas tradicionalmente priorizadas para atendimento odontológico no serviço público, impondo uma discussão sobre o acesso das outras faixas etárias, em especial populações adulta e idosa (NORO et al., 2008).

Em contrapartida, a US NSA apresentou mais pacientes atendidos na faixa etária de 35 a 44 anos, em consonância com estudo que constatou mais uso de serviços odontológicos no grupo etário de 20 a 49 anos. Essa observação justifica-se pela tendência cumulativa das doenças bucais, em especial a cárie dentária, com base nos resultados da Pesquisa Nacional de Saúde Bucal - SB Brasil 2010, em indivíduos de 35-44 anos de idade (CPO-D=16,75), indicando uma possível manutenção da gravidade da cárie na população adulta (BRASIL, 2010). A ausência de uma política de saúde bucal direcionada a essa população pode ser responsável pela manutenção dos altos índices de cárie dental. A falta de acesso a medidas preventivas e assistenciais ou tratamentos pouco resolutivos e de baixa qualidade possivelmente aumentam a complexidade do problema.

O trabalho na APS prevê que as unidades de saúde atuem positivamente no território, realizando cadastramento familiar, diagnóstico situacional e ações dirigidas aos problemas de saúde, buscando o cuidado dos indivíduos e das famílias ao longo do tempo, com postura pró-ativa frente aos problemas de saúde da população. O reconhecimento das micro áreas de risco auxilia na priorização de ações coletivas, com ênfase na prevenção de doenças, direcionadas aos espaços territoriais onde as iniquidades são maiores, o que 
resulta num maior impacto sobre as condições de risco (MALUCELLI et al., 2010; BRASIL, 2010).

A distribuição do número de pacientes atendidos de acordo com as micro áreas mostrou-se semelhante na US NSA. Por outro lado, na US SESC observou-se um predomínio de pacientes provenientes das micro áreas mais vulneráveis e mais próximas à US (SESC1, SESC2 e SESC3). Quando se relacionou micro área ao tipo de consulta na US NSA, predominaram as não programadas na micro área NSA1, mais vulnerável e mais distante da US, e as programadas na micro área NSA3, mais próxima da US. A literatura descreve que populações com baixo nível socioeconômico e baixa escolaridade materna buscam mais os serviços para resolução de situações agudas de saúde. Ainda, sabe-se que grupos populacionais com menores níveis de escolaridade visitam o dentista por problemas de saúde bucal autopercebidos, enquanto indivíduos com maiores níveis de escolaridade o fazem para consultas preventivas ou de acompanhamento (BARCELOS; MONKEN, 2007; CASSAL; DEUS; BAVARESCO, 2011). Essa associação entre micro área e tipo de consulta não foi encontrada na US SESC.

Segundo os resultados do SB Brasil 2010, o Brasil saiu de uma condição de média prevalência de cárie em 2003 para uma condição de baixa prevalência em 2010 (BRASIL, 2010). Mesmo com o declínio dessa doença, foi possível observar que o código referente à cárie dentária foi o motivo predominante dos atendimentos em ambas as US e o sexto entre os problemas e diagnósticos mais frequentes do SSC (GRUPO HOSPITALAR CONCEIÇÃO, 2011), sendo o diagnóstico mais comum entre as consultas programadas na US NSA. Nesse sentido, destaca-se que não somente a oferta de serviços odontológicos de qualidade é um importante fator para ampliar o acesso da população a serviços resolutivos, como também o é a percepção das necessidades de saúde bucal por parte dos indivíduos para que se dê a busca por estes serviços. É mister que se desenvolvam políticas educativas com o objetivo de ampliar o conhecimento da população sobre cuidados preventivos e de orientála na procura por cuidados de saúde (PINHEIRO; TORRES, 2006).

Existe uma relação entre o código referente às doenças da polpa e dos tecidos periapicais e as consultas não programadas nas duas US. Há diversos estudos na literatura que afirmam que a dor oriunda dessas patologias é o motivo mais frequente de procura por serviços de urgências odontológicas (MUNERATO; FIAMINGH; PETRY, 2005; CASSAL; DEUS; BAVARESCO, 2011). A doença cárie, além da perda dental, traz consigo a odontalgia, cujos reflexos podem interferir no comportamento dos indivíduos e afetar suas atividades cotidianas. A oferta de procedimentos não mutiladores no tratamento das patologias pulpares irreversíveis, como, por exemplo, o tratamento endodôntico, poderia contribuir nesse sentido (CASSAL; DEUS; BAVARESCO, 2011). No entanto, o que se verifica, na prática, é uma demanda reprimida de endodontia para atenção secundária, principalmente pela baixa capacidade de oferta de serviços especializados, o que compromete o estabelecimento de adequados sistemas de referência e contra referência em saúde bucal.

Dentre as limitações deste estudo destacam-se: (a) não preenchimento ou preenchimento incompleto dos boletins (ex.: falta de código SIASUS referente ao tipo de consulta); (b) não emissão dos boletins de atendimento previamente à consulta; (c) desatualização do cadastro dos pacientes; (d) dificuldade de padronização no preenchimento do código CID-10, a qual está associada à sua natureza, que tem como objetivo classificar doenças, desconsiderando procedimentos que não preveem diagnósticos (ex.: fratura de restauração); (e) férias de três dos cinco cirurgiõesdentistas na US SESC no mês de julho, refletindo em um número menor de atendimento em comparação aos demais meses avaliados; (f) impossibilidade de extrapolar os resultados pelo curto período de tempo avaliado e pelas peculiaridades de cada território e equipes de saúde. Salienta-se que os três primeiros fatores podem estar relacionados à escassez de profissionais auxiliares e técnico exercendo tarefas administrativas, além da sobrecarga da equipe de saúde bucal, que acumula tarefas assistenciais, organizacionais, de ensino e pesquisa. Ao passo que o quarto fator tem relação com o fato de que a realização de apenas uma oficina de treinamento não foi suficiente para uniformizar o preenchimento dos códigos nos boletins.

A partir dos resultados obtidos, pode-se constatar que a maioria dos pacientes da US NSA era do gênero feminino, da faixa etária 35-44 anos, proveniente da micro área de menor risco e mais próxima da US (NSA3); que o acesso aos serviços odontológicos se deu por meio de consultas programadas, e que o código utilizado de cárie dentária foi o mais frequente entre essas consultas. Também se pode afirmar que houve relação entre consulta não programada, diagnóstico de doenças da polpa e dos tecidos periapicais com a micro área mais vulnerável e mais distante da US (NSA1).

$\mathrm{Na}$ US SESC, a maioria dos pacientes era do gênero feminino, pertencente à faixa etária de $0-11$ anos, oriunda da micro área marcada por extrema desigualdade social e mais próxima da US (SESC2), com maior índice de consultas programadas e maior utilização do código referente a exame odontológico completo/consulta da ação programática odontológica de 0-3 anos. Além disso, existiu relação entre as consultas não programadas e 0 diagnóstico de doenças da polpa e dos tecidos periapicais.

Os sistemas de informação em saúde constituem-se em ferramentas importantes tanto na questão assistencial como em questões administrativas e tem como um de seus objetivos 0 fornecimento de dados epidemiológicos, que servem de base para 0 planejamento e a vigilância em saúde. Com a utilização de sistemas adaptados às realidades das unidades de saúde, os dados referentes ao acesso a determinados serviços, como por exemplo, os da Odontologia, podem ser avaliados, pautando o trabalho na lógica da vigilância em saúde e nos princípios norteadores da APS e do SUS. No entanto, a despeito da importância das informações contidas nesses sistemas, em especial o utilizado nesse estudo, o que se observa é a sua subutilização na área odontológica.

Atenta-se para a possibilidade de ampliar a utilização dos dados sobre os territórios das US, adaptando o acesso aos serviços odontológicos às necessidades da população e priorizando ações dirigidas a subgrupos de risco, de acordo com o princípio da equidade. Entende-se que não basta dispor dos serviços em posições estratégicas e inferir que o acesso da população aos serviços esteja assegurado. É conveniente questionar, constantemente, se eles atingem de fato os segmentos que mais necessitam de atenção.

Nesse sentido, reforça-se a necessidade de maior utilização do sistema de informações para a Odontologia, levando em consideração as características dos territórios, das populações e das equipes de saúde, a fim de readequar a distribuição dos recursos e realizar a gestão da assistência em saúde bucal segundo os princípios da APS. Sugere-se, ainda, o estabelecimento de políticas públicas em saúde bucal que considerem as especificidades das populações e que priorizem aquelas de maior risco e mais vulneráveis aos problemas bucais.

\section{Conclusão}

Em suma, o presente estudo demonstrou que é possível a utilização de informações em saúde para balizar a organização do trabalho das equipes de saúde bucal. No entanto, outros estudos são necessários para ampliar a compreensão do perfil do usuário que utiliza os serviços odontológicos na APS, a fim de que, considerando as particularidades de cada território no planejamento da assistência, este modelo de atenção à saúde consiga atender ao indivíduo e à sua família de forma integral, resolutiva e contínua. 


\section{Referências}

A POLÍTICA Nacional de Saúde Bucal do Brasil: registro de uma conquista histórica. Brasília, 2006. 72p. (Série técnica: desenvolvimento de sistemas e serviços de saúde). Disponível em: $<$ http://dab.saude.gov.br/docs/publicacoes/geral/serie_tecnica_11_por t.pdf>. Acesso em: 13 nov. 2011.

ANDERSEN, R.M. Revisiting the behavioral model and access to medical care: does it matter? J. Health Soc. Behav., Albany, v. 36, no. 1, p. 1-10, Mar. 1995.

BADER, J.; ISMAEL, A.; CLARKSON, J. Evidence-based dentistry and the dental research community. J. Dent. Res., Washington, v. 78, no. 9, p. 1480-1483, Sept. 1999.

BARCELOS, C.; MONKEN, M. Instrumentos para o diagnóstico sóciosanitário no Programa Saúde da Família. In: FONSECA, A.F.; CORBO, A.D'A. (Org.). O território e o processo saúde-doença. Rio de Janeiro: EPSJV: Fiocruz, 2007. [acessado em 13 nov 2011]. Disponível em: <http://www.retsus.fiocruz.br/upload/documentos/territorio_e_o_proce sso_2_livro_1.pdf>. Acesso em: 13 nov. 2011.

BARROS, A.J.D.; BERTOLDI, A.D. Desigualdades na utilização e no acesso a serviços odontológicos: uma avaliação em nível nacional. Ciênc. Saúde Coletiva, Rio de Janeiro, v. 7, n.4, p. 709-717, 2002.

BRASIL. Ministério da Saúde. Divisão Nacional de Saúde Bucal. Pesquisa nacional de saúde bucal 2010: principais resultados. Brasília, 2011. 92p. (Série C: estudos e projetos, 4). Disponível em: $<$ http://189.28.128.100/dab/docs/geral/projeto_sb2010_relatorio_final. pdf>. Acesso em: 13 nov. 2011.

BRASIL. Ministério da Saúde. Saúde da família: uma estratégia para reorganização do modelo assistencial. Brasília, 1997. 36p. Disponível em: <http://bvsms.saude.gov.br/bvs/publicacoes/cd09_16.pdf>. Acesso em: 13 nov. 2011.

CASSAL, J.B.; DEUS, D.C.; BAVARESCO, C.S. Perfil dos usuários de urgência odontológica em uma unidade de atenção primária à saúde. Rev. APS, juiz de Fora, v. 14, n. 1, p. 85-92, jan./mar. 2011. GONDIM, G.M.M. et al. O território da saúde: a organização do sistema de saúde e a territorialização. In: MIRANDA, A.C. et al. (Org.). Território, ambiente e saúde. Rio de Janeiro: Ed. Fiocruz, 2008. p. 237-255.

GRUPO HOSPITALAR CONCEIÇÃO. Sistemas de informações em saúde do serviço de saúde comunitária: indicadores de saúde. Porto Alegre, 2011. 56p.

INSTITUTO BRASILEIRO DE GEOGRAFIA E ESTATÍSTICA. Pesquisa nacional por amostra de domicílios: síntese de indicadores 2008. Disponível em: URL: <http://www.ibge.gov.br/home/estatistica/populacao/trabalhoerendime nto/pnad2008/sintesepnad2008.pdf>. Disponível em: 13 nov. 2010.

LEVCOVITZ, E.; GARRIDO, N.G. Saúde da família: a procura de um modelo enunciado. Cad. Saúde Família, Brasília, v. 1, n. 1, p. 3-15, 1996.

MALUCELLI, A. et al. Classification of risk micro-areas using data mining. Rev. Saúde Pública, São Paulo, v. 44, no. 2, p. 292-300, Apr. 2010.
MATOS, D.L. et al. Projeto Bambuí: avaliação de serviços odontológicos privados, públicos e de sindicato. Rev Saúde Pública, São Paulo, v. 36, no. 2, p. 237-243, Apr. 2002.

MUNERATO, M.C.; FIAMINGH, D.L.; PETRY, P.C. Urgências em odontologia: um estudo retrospectivo. Rev. Fac. Odontol. Porto Alegre, Porto Alegre, v. 46, n. 1, p. 90-95, jul. 2005.

NORO, L.R.A. et al. A utilização de serviços odontológicos entre crianças e fatores associados em Sobral, Ceará, Brasil. Cad. Saúde Pública, São Paulo, v. 24, n. 7, p. 1509-1516, jul. 2008.

PEREIRA, M.G. Serviços de saúde. In: PEREIRA, M. G. Epidemiologia: teoria e prática. Rio de Janeiro: Guanabara Koogan, 2002. p. 513-537.

PINHEIRO, R.S. et al. Gênero, morbidade, acesso e utilização de serviços de saúde no Brasil. Cienc Saúde Coletiva, Rio de Janeiro, v. 7, n. 4, p. 687-707, 2002.

PINHEIRO, R.S.; TORRES, T.Z.G. Uso de serviços odontológicos entre os estados do Brasil. Ciênc. Saúde Coletiva, Rio de Janeiro, v. 11 , n. 4, p. 999-1010, out./dez. 2006.

PRATIS, N. S.; BACCHI, E. O. S. Tratamento ortodôntico da Classe III. R. Gaúcha Odontol., Porto Alegre, v. 37, n. 5, p. 331-34, set./out. 1989.

RODRIGUES, R.P.; FERNANDEZ, R.R.; FUNK, C.S. A padronização do uso da Classificação Internacional de Doenças (CID) no diagnóstico dos motivos de consulta em saúde bucal em duas Unidades de Atenção Primária, Porto Alegre - RS. 2010. Trabalho de Conclusão (Especialização)- Residência Integrada em Saúde, Grupo Hospitalar Conceição, Porto Alegre, 2010.

SAWYER, D.O.; LEITE, I.C.; ALEXANDRINO, R. Perfis de utilização de serviços de saúde no Brasil. Ciênc. Saúde Coletiva, Rio de Janeiro,v. 4, n. 7, p. 757-776, 2002.

SOUZA, T.M.S.; RONCALLI, A.G. Saúde bucal no Programa Saúde da Família: uma avaliação do modelo assistencial. Cad. Saúde Pública, Rio de Janeiro, v. 23, n. 11, p. 2727-39, 2007.

STARFIELD, B. Acessibilidade e primeiro contato: a "porta". In: STARFIELD, B. (Org.). Atenção primária: equilíbrio entre necessidades de saúde, serviços e tecnologia. Brasília: UNESCO/Ministério da Saúde, 2002. p. 207-245.

VERBRUGGE, L.M. The Twain meet: empirial explanations of sex differences in health and mortality. J Health Soc. Behav., Albany, v. 30, p. 282-304, Sept. 1989. 